OPEN ACCESS

Edited by:

Alfredo G. Torres,

University of Texas Medical Branch,

United States

Reviewed by:

Tonya J. Webb,

University of Maryland, Baltimore,

United States

Bindu Sukumaran,

Duke-NUS Medical School, Singapore

*Correspondence:

José De La Fuente jose_delafuente@yahoo.com

Received: 19 June 2017 Accepted: 26 July 2017 Published: 09 August 2017

Citation: Contreras M, Alberdi P, Fernández De Mera IG, Krull C, Nijhof A, Villar M and De La Fuente J (2017) Vaccinomics Approach to the Identification of Candidate Protective Antigens for the Control of Tick Vector Infestations and Anaplasma phagocytophilum Infection.

Front. Cell. Infect. Microbiol. 7:360 doi: 10.3389/fcimb.2017.00360

\section{Vaccinomics Approach to the} Identification of Candidate Protective Antigens for the Control of Tick Vector Infestations and Anaplasma phagocytophilum Infection

\author{
Marinela Contreras ${ }^{1}$, Pilar Alberdi ${ }^{1}$, Isabel G. Fernández De Mera ${ }^{1}$, Christoph Krull ${ }^{2}$, \\ Ard Nijhof ${ }^{2}$, Margarita Villar ${ }^{1}$ and José De La Fuente ${ }^{1,3 *}$
}

${ }^{1}$ SaBio, Instituto de Investigación en Recursos Cinegéticos IREC-CSIC-UCLM-JCCM, Ciudad Real, Spain, ${ }^{2}$ Institute for Parasitology and Tropical Veterinary Medicine, Freie Universität Berlin, Berlin, Germany, ${ }^{3}$ Department of Veterinary Pathobiology, Center for Veterinary Health Sciences, Oklahoma State University, Stillwater, OK, United States

Anaplasma phagocytophilum is an emerging tick-borne pathogen causing human granulocytic anaplasmosis (HGA), tick-borne fever (TBF) in small ruminants, and other forms of anaplasmosis in different domestic and wild animals. The main vectors of this pathogen are Ixodes tick species, particularly I. scapularis in the United States and $I$. ricinus in Europe. One of the main limitations for the development of effective vaccines for the prevention and control of $A$. phagocytophilum infection and transmission is the identification of effective tick protective antigens. The objective of this study was to apply a vaccinomics approach to I. scapularis-A. phagocytophilum interactions for the identification and characterization of candidate tick protective antigens for the control of vector infestations and $A$. phagocytophilum infection. The vaccinomics pipeline included the use of quantitative transcriptomics and proteomics data from uninfected and $A$. phagocytophilum-infected $I$. scapularis ticks for the selection of candidate protective antigens based on the variation in tick mRNA and protein levels in response to infection, their putative biological function, and the effect of antibodies against these proteins on tick cell apoptosis and pathogen infection. The characterization of selected candidate tick protective antigens included the identification and characterization of 1. ricinus homologs, functional characterization by different methodologies including RNA interference, immunofluorescence, gene expression profiling, and artificial tick feeding on rabbit antibodies against the recombinant antigens to select the candidates for vaccination trials. The vaccinomics pipeline developed in this study resulted in the identification of two candidate tick protective antigens that could be selected for future vaccination trials. The results showed that I. scapularis lipocalin (ISCW005600) and lectin pathway inhibitor (AAY66632) and I. ricinus homologs constitute candidate protective antigens for the control of vector infestations and $A$. phagocytophilum infection. Both antigens are involved in the tick evasion of host defense response and pathogen infection and transmission, but targeting different immune response pathways. The vaccinomics 
pipeline proposed here could be used to continue the identification and characterization of candidate tick protective antigens for the development of effective vaccines for the prevention and control of HGA, TBF, and other forms of anaplasmosis caused by A. phagocytophilum.

Keywords: anaplasmosis, immunology, vaccine, tick, Ixodes, Anaplasma phagocytophilum

\section{INTRODUCTION}

The intracellular bacterium, Anaplasma phagocytophilum (Rickettsiales: Anaplasmataceae) is an emerging tick-borne pathogen causing human granulocytic anaplasmosis (HGA), which has emerged as a tick-borne disease of humans in the United States, Europe and Asia, and tick-borne fever (TBF) in small ruminants, most notably in sheep in Europe (Gordon et al., 1932; Foggie, 1951; Dumler et al., 2001; Stuen et al., 2013; Bakken and Dumler, 2015; Dugat et al., 2015; Severo et al., 2015). Clinical presentation of $A$. phagocytophilum infection has been also documented in goats, cattle, horses, dogs, cats, roe deer, and reindeer (Severo et al., 2015). The main vectors of this pathogen are Ixodes tick species, particularly $I$. scapularis in the United States and I. ricinus in Europe (Stuen et al., 2013; Bakken and Dumler, 2015).

Despite the burden that $A$. phagocytophilum represents for humans and animals, vaccines are not available for prevention and control of pathogen infection and transmission (Dumler et al., 2001; Stuen et al., 2013, 2015; Bakken and Dumler, 2015; Severo et al., 2015; Contreras et al., 2017). One of the main limitations for the development of effective vaccines for the prevention and control of $A$. phagocytophilum infection and transmission is the identification of effective tick protective antigens. Recently, different approaches have been developed for the identification and characterization of candidate tick protective antigens (de la Fuente and Contreras, 2015; de la Fuente et al., 2016a). Vaccinomics is one of the approaches that have been used by our group for the identification of tickderived and pathogen-derived protective antigens (de la Fuente and Merino, 2013; Merino et al., 2013; Antunes et al., 2014; de la Fuente and Contreras, 2015; Contreras et al., 2016, 2017; de la Fuente et al., 2016a; Villar et al., 2017). Vaccinomics is a holistic approach based on the use of genome-scale or omics technologies integrated in a systems biology approach to characterize tick-host-pathogen interactions for the development of next-generation vaccines (de la Fuente and Merino, 2013; Contreras et al., 2016; de la Fuente et al., 2016a; Villar et al., 2017). In this translational approach, basic biological information on tick-host-pathogen interactions translates into the identification and subsequent evaluation of new candidate protective antigens (de la Fuente and Merino, 2013; de la Fuente et al., 2016a; Villar et al., 2017).

The sequence, assembly and annotation of the I. scapularis genome were recently released (Gulia-Nuss et al., 2016), and various genomics, transcriptomics and proteomics studies in $I$. ricinus suggest that these tick species are genetically closely related (Schwarz et al., 2013, 2014; Genomic Resources Development Consortium et al., 2014; Cramaro et al., 2015;
Kotsyfakis et al., 2015; Weisheit et al., 2015; Chmelař et al., 2016). These results open new opportunities for research on tickhost-pathogen interactions and the possibility of identifying tick protective antigens for both $I$. scapularis and I. ricinus major vectors of $A$. phagocytophilum (de la Fuente et al., 2016b).

Recently, transcriptomics, proteomics and metabolomics datasets have been integrated and used for the characterization of I. scapularis-A. phagocytophilum molecular interactions (Ayllón et al., 2015; Villar et al., 2015a,b, 2016; Cabezas-Cruz et al., 2016, 2017a,b; de la Fuente et al., 2016c, 2017; Gulia-Nuss et al., 2016; Shaw et al., 2017). Herein, a vaccinomics pipeline was developed based on quantitative transcriptomics and proteomics data from uninfected and A. phagocytophilum-infected I. scapularis nymphs, adult female midguts and salivary glands, and ISE6 cells (Ayllón et al., 2015; Villar et al., 2015a). The vaccinomics pipeline was then used for the identification of candidate protective antigens for the control of vector infestations and pathogen infection. The results showed that I. scapularis ISCW005600 and AAY66632 and $I$. ricinus homologs constitute candidate protective antigens for the control of vector infestations and $A$. phagocytophilum infection.

\section{MATERIALS AND METHODS}

\section{Ticks and Cultured Tick Cells}

Ixodes scapularis ticks were obtained from the laboratory colony maintained at the Oklahoma State University Tick Rearing Facility. Nymphs and adult female I. scapularis were infected with $A$. phagocytophilum by feeding on a sheep inoculated intravenously with approximately $1 \times 10^{7} \mathrm{~A}$. phagocytophilum (NY18 isolate)-infected HL-60 human cells (90-100\% infected cells) (Kocan et al., 2012; Ayllón et al., 2015). Animals were housed and experiments conducted with the approval and supervision of the OSU Institutional Animal Care and Use Committee (Animal Care and Use Protocol, ACUP No. VM1026). I. ricinus ticks were obtained from the laboratory colony maintained at the Freie Universität Berlin. Larvae and nymphs were fed on mice and adults on rabbits. The $I$. scapularis embryo-derived tick cell line ISE6, provided by Ulrike Munderloh, University of Minnesota, USA, was cultured in L15B300 medium as described previously (Kurtti et al., 1996; Munderloh et al., 1999; Villar et al., 2015a). IRE/CTVM20 embryo-derived tick cells, provided by the Tick Cell Biobank, were maintained as described previously (Bell-Sakyi et al., 2007; Alberdi et al., 2015). Tick cells were first inoculated with A. phagocytophilum (human NY18 isolate; Asanovich et al., 1997)-infected HL-60 cells and maintained according to Munderloh et al. (1999). Uninfected and infected cultures $(N=4$ independent cultures with approximately $10^{7}$ cells each) were 
sampled at 7 days post-infection (dpi) (75\% infected cells). The percentage of cells infected with $A$. phagocytophilum was calculated by examining at least 200 cells using a $100 x$ oil immersion objective.

\section{Transcriptomics and Proteomics Datasets}

The quantitative transcriptomics and proteomics data for uninfected and A. phagocytophilum-infected I. scapularis nymphs, adult female midguts and salivary glands, and ISE6 cells were obtained from previously published results (Ayllón et al., 2015; Villar et al., 2015a) and deposited at the Dryad repository database, NCBI's Gene Expression Omnibus database and ProteomeXchange Consortium via the PRIDE partner repository with the dataset identifier PXD002181 and doi: 10.6019/PXD002181.

\section{Sequence Analysis}

To find the I. ricinus homologs, selected $I$. scapularis sequences were blasted against the I. ricinus database using the Blastp tool from BLAST (Altschul et al., 1990; Madden et al., 1996), and the sequences with the lowest $E$-value were selected. Gene ontology (GO) analysis for biological process (BP) was done with Blast2GO software (version 3.0; http://www.blast2go.com) (Villar et al., 2014).

\section{Production of Recombinant Proteins}

The coding sequences for I. scapularis candidate protective antigens were amplified from synthetic genes optimized for codon usage in Escherichia coli (Genscript Corporation, Piscataway, NJ, USA) using sequence-specific primers (Table 1). The amplified DNA fragments were cloned into the expression vector pET101 and expressed in E. coli strain BL21 using the Champion pET101 Directional TOPO Expression kit (Carlsbad, CA, USA). Recombinant proteins were fused to Histidine tags for purification by affinity to Ni (Merino et al., 2013; Moreno-Cid et al., 2013). Transformed E. coli strains were induced with IPTG for $4.5 \mathrm{~h}$ to produce recombinant proteins, which were purified to $>85 \%$ of total cell proteins by $\mathrm{Ni}$ affinity chromatography (Genscript Corporation) as previously described (Merino et al., 2013; Moreno-Cid et al., 2013) using $1 \mathrm{ml}$ HisTrap FF columns mounted on an AKTA-FPLC system (GE Healthcare, Piscataway, NJ, USA) in the presence of $7 \mathrm{M}$ urea lysis buffer. The purified antigens were refolded by dialysis against 1,000 volumes of PBS, pH 7.4 (137 mM NaCl, $2.7 \mathrm{mM} \mathrm{KCl,} 10 \mathrm{mM} \mathrm{Na} 2 \mathrm{HPO}_{4}, 1.8 \mathrm{mM}$ $\mathrm{KH}_{2} \mathrm{PO}_{4}$ ) for $12 \mathrm{~h}$ at $4^{\circ} \mathrm{C}$.

\section{Production of Rabbit Polyclonal IgG Antibodies}

For each recombinant tick protein and total ISE6 tick cell proteins, two New Zealand white rabbits (Oryctulagus cuniculus) were subcutaneously injected at weeks 0,4 , and 6 with $50 \mu \mathrm{g}$ protein in $0.4 \mathrm{ml}$ Montanide ISA $50 \mathrm{~V}$ adjuvant (Seppic, Paris, France). Blood was collected before injection and 2 weeks after the last immunization to prepare pre-immune and immune sera, respectively. Serum aliquots were kept at $4^{\circ} \mathrm{C}$ for immediate use or at $-20^{\circ} \mathrm{C}$ for long-term storage. The IgG were purified from serum samples using the Montage antibody purification kit and
TABLE 1 | Oligonucleotide primers used in this study for cloning, RNAi and RT-PCR.

\begin{tabular}{ll}
\hline GenBank accession No. & $\begin{array}{l}\text { Oligonucleotide sequence }\left(5^{\prime}-3^{\prime} \text { for forward }\right. \\
\text { and reverse primers) }\end{array}$
\end{tabular}

\section{CLONING}

ISCW024685

CACCATGAAAAGCAGCGCACTGCTG

ISCW024295

GCGTTACCACGAACGCACC

ISCW022212

CACCATGCCGAAACAAGGCGAAAC

(S)

TCCAGAGTCACCACACAAAACG

ISCW020900

CACCATGTGGGGTCAGATTGCGCT

ACAGATGAATIITCAGGC

ISCW000326

CACCATGAACAAAGCGATCTTCAT

CACTTCACCGAAAAAGCCGC

ISCW008146

CACCATGCCGGCGTCAATGAAAAG

CAGAGAACCCAGATTCGGAA

CACCATGGATITGATGACCTGTT

GAAGCTCAGGGTGTTCTGTT

ISCW008641

CACCATGCAACGTGACATIITAG

CCAACAGCCCGGCTGCGATT

ISCW024499

CACCATGTGCCTGGTGTITGCAAC

GCGCAGAAAGGAACTCGTAC

ISCW005600

CACCATGATTCGTCAGGTTCGCGA

CGAACCTGAGATCGATGAGG

ISCW013709

CACCATGTITCGTACCAGCTCTGG

CACAATATAATCCGGTGCAC

ISCW017117

CACCATGCTGAGTGTGCTGCTGGG

CGTGGTGGCGTCCGGCGGCG

ISCW013574

CACCATGTATCAGCTGCGCGATIT

GCAACGGGATTTGCGAACAC

ISCW018900 CACCATGGGCCCGTITATTGGTCT

GCCGATAATGCGACCGATAA

ISCW023907 CACCATGCCGGTCAATCGCCTGAT

AACTITACGAAAGAAAAACA

ISCW024682 CACCATGATTCATGAACCGGTGAT

ATACGGACAGTACAGTITGCA

ISCW015453 CACCATGATGAAAAGCCCGCTGTITAT

ACCGAAAAAGCCGTGGCCGA

ISCW021670

CACCATGTGGGAACTGCATGCCGA

CTCCTGGGTAATATTACGCGT

ISCW017271

CACCATGTGCAGCGATTCTAAACC

CGGCAGATAGGAACCGTGCG

AAY66632

CACCATGGGCCTGACCGGTACCAC

GTTGTCTIGGTITCTGG

\section{RNAi AND RT-PCR}

ISCW005600

AAY66632

DQ066214 (rpS4)
TCCCCTTCTCAAAGGAGGAT*

ATCCACAGGCGGATATGAAG*

ACCCGTTCATGGGACAAATA*

TTCTTGGGCTTCTCAGTTGG*

GGTGAAGAAGATTGTCAAGCAGAG

TGAAGCCAGCAGGGTAGTITG
*The same oligonucleotide primers were used to determine gene expression levels by RT-PCR and for the generation of dsRNA for RNAi. To produce dsRNA, the T7 promoter sequence 5'-GAATTAATACGACTCACTATAGGGAGA-3' was added to the 5'-end of each primer. 
spin columns with PROSEP-A media (Millipore, Billerica, MA, USA) following the manufacturer's recommendations.

\section{Western Blot Analysis}

Ten micrograms of each recombinant protein or $20 \mu \mathrm{g}$ total proteins from ISE6 tick cells were loaded onto a 12\% SDSpolyacrylamide pre-cast gel (Life Science, Hercules, CA, USA) and transferred to a nitrocellulose membrane. The membrane was blocked with $5 \%$ bovine serum albumin (BSA) (SigmaAldrich, St. Louis, MI, USA) for $2 \mathrm{~h}$ at room temperature (RT), and washed four times with TBS (50 mM Tris-Cl, pH 7.5, $150 \mathrm{mM}$ $\mathrm{NaCl}, 0.5 \%$ Tween 20). Purified rabbit IgG were used at a 1:500 dilution in TBS, and the membrane was incubated overnight at $4{ }^{\circ} \mathrm{C}$ and washed four times with TBS. The membrane was then incubated with an anti-rabbit IgG-horseradish peroxidase (HRP) conjugate (Sigma-Aldrich) diluted 1:1,000 in TBS with 3\% BSA. The membrane was washed five times with TBS and finally developed with TMB (3,3', 5,5' - tetramethylbenzidine) stabilized substrate for HRP (Promega, Madrid, Spain) according to the manufacturer recommendations.

\section{Immunofluorescence Assay (IFA) in Adult Female Ticks}

Adult I. scapularis females were infected with A. phagocytophilum (NY18) as described above. Female ticks were removed from the sheep 10 days after infestation, held in the humidity chamber for 4 days and fixed with $4 \%$ paraformaldehyde in $0.2 \mathrm{M}$ sodium cacodylate buffer, dehydrated in a graded series of ethanol and embedded in paraffin (Ayllón et al., 2015). Sections ( $4 \mu \mathrm{m})$ were prepared and mounted on glass slides. The paraffin was removed from the sections with xylene and the sections were hydrated by successive 2 min washes with a graded series of 100, 95, 80, 75 , and $50 \%$ ethanol. The slides were treated with Proteinase K (Dako, Barcelona, Spain) for $7 \mathrm{~min}$, washed with PBS and incubated with 3\% BSA (Sigma-Aldrich) in PBS for $1 \mathrm{~h}$ at RT. The slides were then incubated for $14 \mathrm{~h}$ at $4^{\circ} \mathrm{C}$ with primary rabbit IgG antibodies diluted 1:100 in 3\% BSA/PBS and, after 3 washes in PBS, developed for $1 \mathrm{~h}$ with goat-anti-rabbit IgG conjugated with phycoerythrin (PE) (Sigma-Aldrich) (diluted 1:50 in 3\% BSA/PBS). The slides were washed twice with PBS and mounted in ProLong Antifade with DAPI reagent (Molecular Probes, Eugene, OR, USA). The sections were examined using a Zeiss LSM 800 laser scanning confocal microscope (Carl Zeiss, Oberkochen, Germany). Sections of uninfected ticks and IgG from pre-immune and anti-ISE6 sera were used as controls.

\section{Antibody Inhibition Assay}

The inhibitory effect of rabbit IgG antibodies on $A$. phagocytophilum (NY18) was conducted as described previously (Villar et al., 2015b). ISE6 and IRE/CTVM20 tick cells were pooled and used to seed 24-well plates for each assay. Each well received $1 \times 10^{6}$ cells in L-15B300 (ISE6) or L-15/L-15B (IRE/CTVM20) medium $24 \mathrm{~h}$ prior to inoculation with $A$. phagocytophilum. Infected cultures for inoculum were harvested when infection reached $80 \%$ and host cells were mechanically disrupted with a syringe and 26-gauge needle. Purified IgG $(100 \mu \mathrm{g} / \mathrm{ml})$ were added to the culture media and incubated with the cells for $48 \mathrm{~h}$. Then, the medium with antibodies was removed and the $A$. phagocytophilum inoculum $(100 \mu \mathrm{l})$ was added to the cell monolayers and incubated at $31^{\circ} \mathrm{C}$ for $60 \mathrm{~min}$. The inoculum was removed from the wells and cell monolayers washed three times with PBS. Complete medium $(1 \mathrm{ml})$ was added to each well and the plates were incubated at $31^{\circ} \mathrm{C}$. The control included inoculum incubated with rabbit pre-immune and anti-ISE6 IgG. Four replicates were done for each treatment. After $72 \mathrm{~h}$, cells from all wells were harvested and processed for A. phagocytophilum detection by real-time PCR after DNA extraction. Results were compared between treatments by the Student's $t$-test with unequal variance $(P=0.05 ; N=4$ biological replicates).

\section{Flow Cytometry of Tick Cells Incubated with Rabbit IgG Antibodies}

Approximately $5 \times 10^{5}-1 \times 10^{6}$ of $A$. phagocytophilum-infected ISE6 and IRE/CTVM20 tick cells were collected after incubation with rabbit IgG. Purified IgG $(2.2-2.4 \mathrm{mg} / \mathrm{ml})$ were mixed with $A$. phagocytophilum and incubated with tick cells as described above in the antibody inhibition assay. Apoptosis was measured by flow cytometry using the Annexin V-fluorescein isothiocyanate (FITC) apoptosis detection kit (Immunostep, Salamanca, Spain) following the manufacturer's protocols. The technique detects changes in phospholipid symmetry analyzed by measuring Annexin V (labeled with FITC) binding to phosphatidylserine, which is exposed in the external surface of the cell membrane in apoptotic cells. Cells were stained simultaneously with the non-vital dye propidium iodide (PI) allowing the discrimination of intact cells (Annexin V-FITC negative, PI negative) and early apoptotic cells (Annexin V-FITC positive, PI negative). All samples were analyzed on a FAC-Scalibur flow cytometer equipped with CellQuest Pro software (BD Biosciences, Madrid, Spain). The viable cell population was gated according to forward-scatter and side-scatter parameters. The percentage of apoptotic cells was determined by flow cytometry after Annexin V-FITC and PI labeling and compared between treated and untreated uninfected cells by Student's $t$-test with unequal variance $(P=0.05 ; N=4$ biological replicates).

\section{RNA Interference (RNAi) for Gene Knockdown in Tick Cells}

RNAi was used to characterize the effect of gene knockdown on tick cell pathogen infection. Oligonucleotide primers homologous to selected I. scapularis ISCW005600 and AAY66632 genes containing T7 promoters (Table 1) were used for in vitro transcription and synthesis of dsRNA as described previously (Ayllón et al., 2013), using the Access RT-PCR system (Promega, Madison, WI, USA) and the Megascript RNAi kit (Ambion, Austin, TX, USA). The unrelated Rs 86 dsRNA was synthesized using the same methods described previously and used as negative control (Ayllón et al., 2013). The dsRNA was purified and quantified by spectrophotometry. RNAi experiments were conducted in cell cultures by incubating ISE6 tick cells with $10 \mu \mathrm{l}$ dsRNA $\left(5 \times 10^{10}-5 \times 10^{11}\right.$ molecules/ $\left.\mu \mathrm{l}\right)$ and $90 \mu \mathrm{L}$ L15B300 medium in 24-well plates using 5 wells per treatment (Ayllón 
et al., 2013). Control cells were incubated with the unrelated Rs 86 dsRNA. After $48 \mathrm{~h}$ of dsRNA exposure, tick cells were infected with cell-free A. phagocytophilum (NY18) obtained from approximately $5 \times 10^{6}$ infected HL-60 cells (90-100\% infected cells) (Thomas and Fikrig, 2007) and resuspended in culture medium to use $1 \mathrm{ml} /$ well. Cells were incubated for an additional $72 \mathrm{~h}$, harvested and used for DNA and RNA extraction. RNA was used to analyze gene knockdown by real-time RT-PCR with respect to Rs86 control. DNA was used to quantify the $A$. phagocytophilum infection levels by real-time PCR.

\section{Determination of $A$. phagocytophilum Infection by Real-Time PCR}

A. phagocytophilum DNA levels were characterized by major surface protein 4 (msp4) real-time PCR normalized against tick ribosomal protein S4 (rpS4) as described previously (Ayllón et al., 2015). Normalized Ct-values were compared between untreated and treated cells by Student's $t$-test with unequal variance $(P=$ $0.05 ; N=4$ biological replicates).

\section{Determination of Tick mRNA Levels by Real-Time RT-PCR}

Total RNA was extracted from ISE6 tick cell cultures using TriReagent (Sigma-Aldrich) following manufacturer's recommendations. The expression of selected I. scapularis ISCW005600 and AAY66632 genes was characterized using total RNA extracted from infected and uninfected ISE6 tick cells. Real-time RT-PCR was performed on RNA samples using genespecific oligonucleotide primers (Table 1) and the Kapa SYBR Fast One-Step qRT-PCR Kit (Kapa Biosystems, Wilmington, MA, USA) and the Rotor-Gene Real-Time PCR Detection System (Qiagen, Madrid, Spain). A dissociation curve was run at the end of the reaction to ensure that only one amplicon was formed and that the amplicons denatured consistently at the same temperature range for every sample. The mRNA levels were normalized against tick $r p S 4$ using the genNorm method (Delta-Delta-Ct, ddCT) as described previously (Ayllón et al., 2015). Normalized Ct-values were compared between infected and uninfected tick cells by Student's $t$-test with unequal variance ( $P=0.05 ; N=4$ biological replicates).

\section{Artificial Tick Feeding}

Artificial tick feeding was conducted as previously described for Dermacentor reticulatus (Krull et al., 2017). Briefly, 1719 female and 3 male I. ricinus ticks were placed on each feeding unit. The feeding unit was subsequently closed by the insertion of a pierced plastic lid (PE-LD Stopfen $26 \mathrm{~mm}$, Brimon Laborbedarf, Hamburg, Germany) wrapped in gauze fabric into the feeding unit, leaving approximately one $\mathrm{cm}$ between the silicone membrane and lid. The feeding unit was then hung into a glass beaker ( $50 \mathrm{ml}$, Simax, Czech Republic) containing the bovine blood using a rubber ring with an inner diameter of $32 \mathrm{~mm}$ (Lux, Wermelskirchen, Germany). Blood was supplemented with ATP and gentamycin (Krull et al., 2017), and $5 \mathrm{ml}$ blood per feeding unit was pipetted into a sterile beaker and preheated to $37^{\circ} \mathrm{C}$ on a hot plate. The blood was changed twice daily at $12 \pm 2 \mathrm{~h}$ intervals. During each blood change, the outside of the feeding unit and underside of the silicone membrane were rinsed with sterile $0.9 \% \mathrm{NaCl}$ solution, preheated to body temperature. The number of attached, dead and fed ticks was counted after which the feeding unit was transferred to a new sterile beaker with fresh blood. Males stayed inside the feeding unit until the end of the experiment, to provide them with sufficient opportunity and time to fertilize any females present. Feeding units were placed in an incubator (ICH 256C, Memmert $\mathrm{GmbH}$, Schwabach, Germany), where the blood was maintained at a constant temperature of $37^{\circ} \mathrm{C}$ using a heating plate (Hot Plate 062, Labotect, Göttingen, Germany). Environmental conditions were set at $20^{\circ} \mathrm{C}, 80 \%$ relative humidity, $5 \% \mathrm{CO}_{2}$ and $15 \mathrm{~h}$ light $/ 9$ $\mathrm{h}$ dark. Once ticks were partially engorged, the feeding units were transferred to a six-well plate (Sarstedt, Nümbrecht, Germany) and ticks were fed for $36 \mathrm{~h}$ with $3 \mathrm{ml}$ blood supplemented with $1 \mathrm{mg} / \mathrm{ml}$ of pre-immune or antigen-specific purified IgG. Dead and detached engorged ticks were removed, and engorged females that detached were weighed and stored individually in $2 \mathrm{ml}$ Eppendorf tubes with pierced lids, which were kept in desiccators with approximately $90 \%$ relative humidity at RT. Ticks were assessed for egg mass 8 weeks post-feeding. The number of dead/fed ticks, ticks and eggs weight, and ticks with or without oviposition were compared between groups by a Fisher's exact test $(P=0.05$; http://www.socscistatistics.com/tests/fisher/ Default2.aspx).

\section{RESULTS AND DISCUSSION}

\section{Selection of Candidate Tick Protective Antigens}

A vaccinomics pipeline was developed for the selection and characterization of candidate tick protective antigens for the control of vector infestations and pathogen infection (Figure 1). The vaccinomics pipeline included the use of quantitative transcriptomics and proteomics data from uninfected and A. phagocytophilum-infected I. scapularis ticks (Ayllón et al., 2015) for the selection of candidate protective antigens based on the variation in tick mRNA and protein levels in response to infection, their putative biological function, and the effect of antibodies against these proteins on tick cell apoptosis and pathogen infection (Figure 1). The characterization of selected candidate tick protective antigens included the identification and characterization of I. ricinus homologs, functional characterization by different methodologies including RNAi, IFA, gene expression profile, and artificial tick feeding on rabbit antibodies against the recombinant antigens to select the candidates for vaccination trials (Figure 1). This process could be repeated as many times as needed to cover all potential candidate antigens or until the desired number of candidate antigens for vaccination trials is reached (Figure 1). The vaccinomics pipeline included some of the algorithms previously proposed (de la Fuente and Merino, 2013; Contreras et al., 2016) and validated (Merino et al., 2013; Antunes et al., 2014) for the selection and characterization of candidate protective antigens, but for the first time it was applied to integrated transcriptomics and proteomics data of tick-pathogen interactions. 


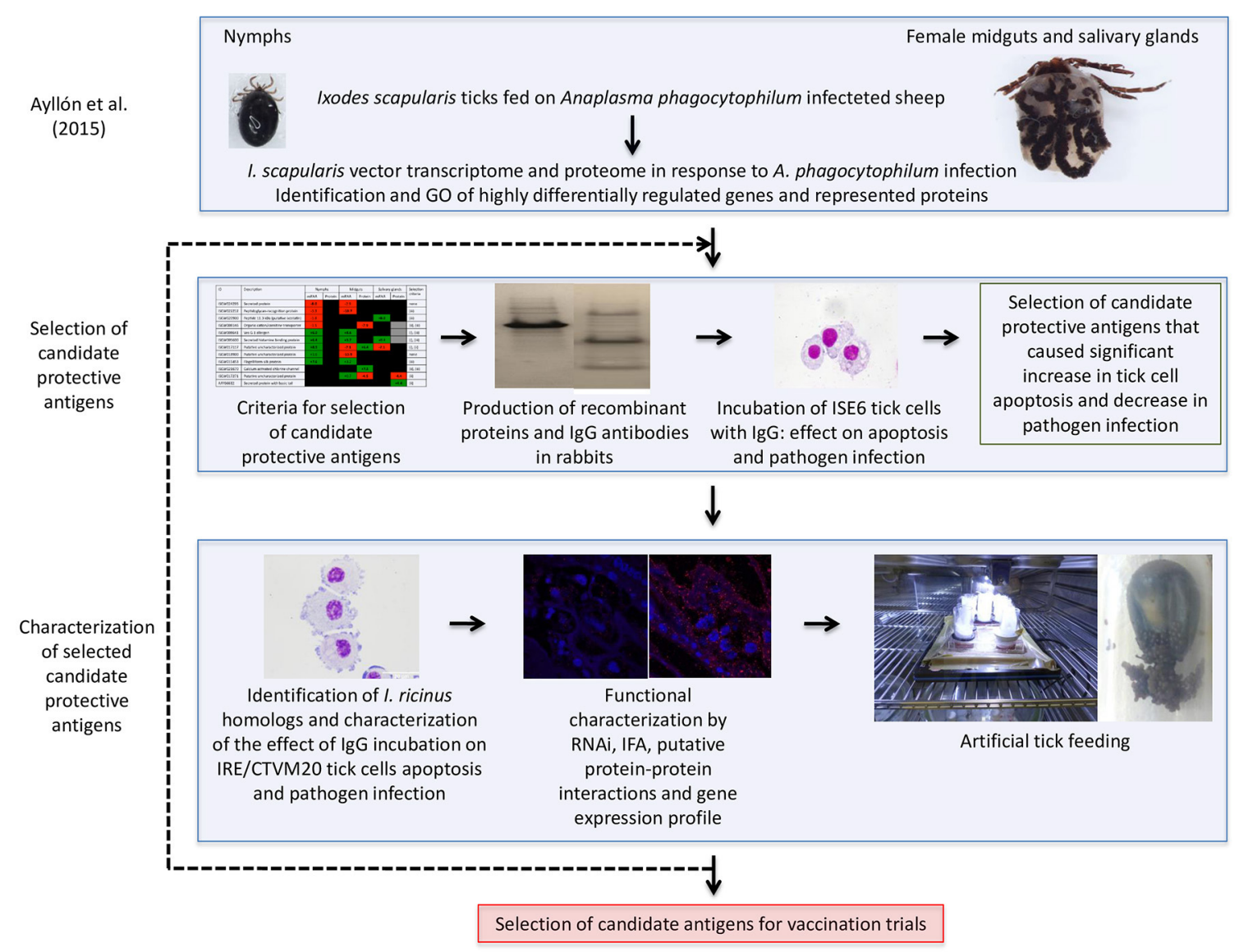

FIGURE 1 | Vaccinomics pipeline for the selection and characterization of candidate tick protective antigens for the control of vector infestations and pathogen infection. The quantitative transcriptomics and proteomics data for uninfected and $A$. phagocytophilum-infected I. scapularis nymphs, adult female midguts and salivary glands were obtained from previously published results (Ayllón et al., 2015). The selection of candidate tick protective antigens included criteria based on the variation in tick mRNA and protein levels in response to infection, their putative biological function, and the effect of antibodies against these proteins on tick cell apoptosis and pathogen infection. The characterization of selected candidate protective antigens included the identification and characterization of I. ricinus homologs, functional analyses by different methodologies, and artificial tick feeding on rabbit antibodies against the recombinant antigens to select the candidates for vaccination trials.

The characterization of tick-pathogen molecular interactions was based on the previous work by Ayllón et al. (2015) of the I. scapularis transcriptome and proteome in response to $A$. phagocytophilum infection in nymphs and female midguts and salivary glands. The highly differentially regulated genes were selected as those with more than 50 -fold ( $\log 2$ normalized fold change $>5.64$ ) difference between infected and uninfected tick samples $(P<0.00003)$ (Figure 2A). The highly differentially represented proteins were selected as those with more than 15 -fold (log2 normalized fold change $>3.90$ ) change between infected and uninfected tick samples $(P<0.00003)$ (Figure 2A). Of the highly differentially regulated/represented genes/proteins, between 0 and 50\% were identified at both mRNA and protein levels in the different samples (Figure 2B). The analysis of highly differentially expressed/represented genes/proteins in response to $A$. phagocytophilum infection evidenced tissuespecific differences in response to infection (Ayllón et al., 2015), which were taken into consideration for the selection of candidate protective antigens (Figures 2A-D). The candidate protective antigens were selected by using the criteria (i) highly differentially up-regulated genes in at least two samples, (ii) highly down-regulated genes in at least one sample, (iii) highly differentially over-represented proteins and identified in the $I$. scapularis proteome, (iv) highly differentially under-represented proteins and identified in the I. scapularis proteome, and/or (v) putative BP in tick-pathogen and tick-host interactions (Figure 3A). The rationale behind the selection criteria for candidate protective antigens was based on their putative relevance in (i, iii) tick response to infection (de la Fuente et al., 2016c,d, 2017), (ii, iv) manipulated by A. phagocytophilum to decrease tick protective mechanisms and increase infection (de la Fuente et al., 2016c,d, 2017), and (v) tick-pathogen and tick-host interactions (Figure 3B).

By using these criteria, a total of 12 candidate tick protective antigens were initially selected, and 7 of them fulfilled two of the selection criteria (Figure 3A). The recombinant antigens were 


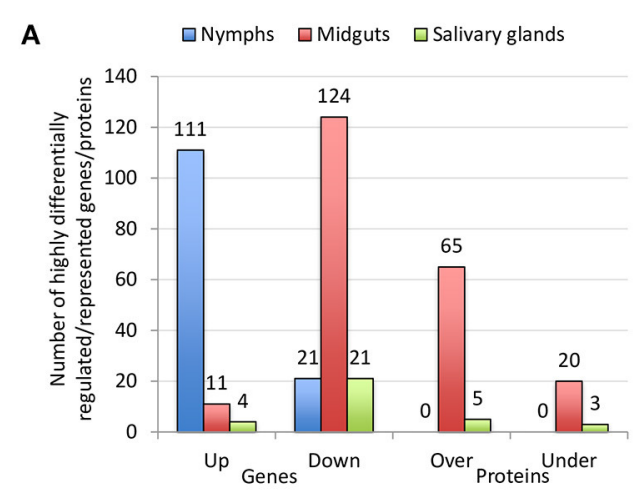

C

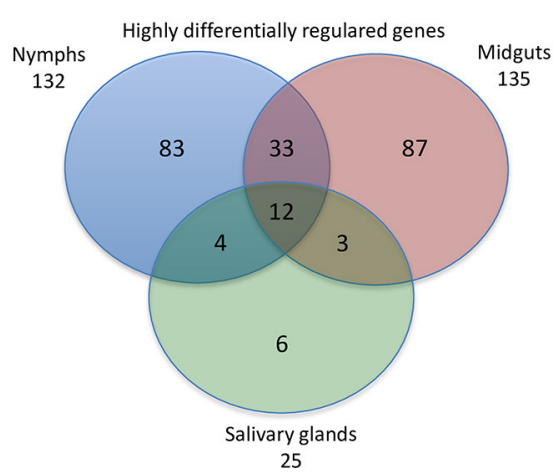

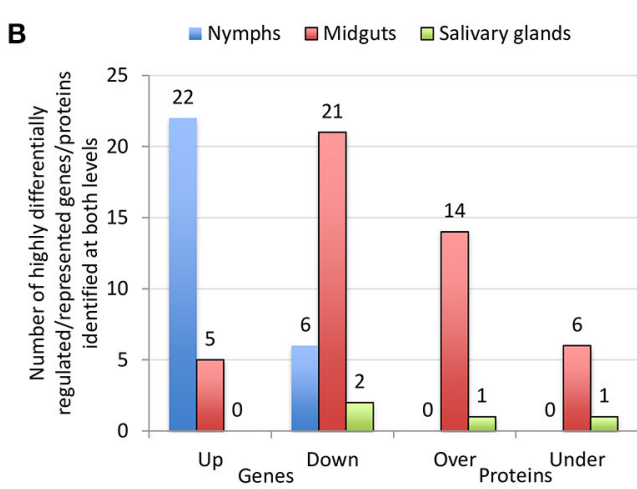

D

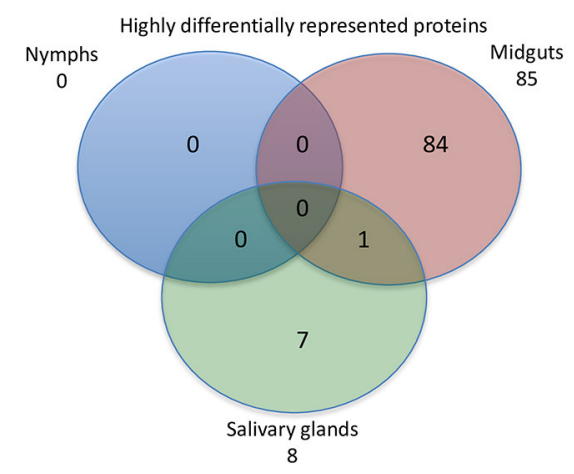

FIGURE 2 | Effect of $A$. phagocytophilum infection on I. scapularis highly differentially regulated genes and represented proteins. (A) Number of tick highly differentially regulated genes and differentially represented proteins that were up-regulated (Up), down-regulated (Down), over-represented (Over) and under-represented (Under) in response to pathogen infection. (B) Number of tick highly differentially regulated genes and differentially represented proteins that were up-regulated (Up),

down-regulated (Down), over-represented (Over) and under-represented (Under) in response to pathogen infection, and identified at both mRNA and protein levels. (C) Venn diagram with highly differentially regulated genes that were identified in tick nymphs, midguts and salivary glands. (D) Venn diagram with highly differentially represented proteins that were identified in tick nymphs, midguts and salivary glands. The highly differentially regulated genes were selected as those with more than 50 -fold (log2 normalized fold change $>5.64$ ) difference between infected and uninfected tick samples $(P<0.00003)$. The highly differentially represented proteins were selected as those with more than 15 -fold (log2 normalized fold change $>3.90$ ) change between infected and uninfected tick samples $(P<0.00003)$. The quantitative transcriptomics and proteomics data for uninfected and $A$. phagocytophilum-infected $I$. scapularis nymphs, adult female midguts and salivary glands were obtained from previously published results (Ayllón et al., 2015).

produced in E. coli and used for the preparation of antigenspecific IgG antibodies in immunized rabbits (Figures 4A,B). These IgG antibodies were then used for the incubation with $I$. scapularis ISE6 cells before infection with A. phagocytophilum to characterize the effect on cell apoptosis (Figure 5A) and pathogen infection (Figure 5B). The results showed that anti ISCW005600 and AAY66632 IgG significantly increased the percentage of apoptotic cells when compared to negative control cells incubated with pre-immune IgG (Figure 5A). The incubation of ISE6 cells with rabbit IgG against recombinant antigens significantly decreased pathogen infection for 7 antigens when compared to the negative control (Figure 5B). The positive control cells were incubated with rabbit IgG antibodies against total ISE6 tick cells proteins, which significantly increased cell apoptosis but did not affect pathogen infection when compared to the negative control (Figures 5A,B). The anti-ISE6 antibodies did not affect pathogen infection of tick cells, which as previously discussed (Stuen et al., 2015) was due to the presence of not protective dominant antigens in the protein extract used to immunize rabbits for antibody production. Nevertheless, these results showed that incubation of ISE6 tick cells with IgG antibodies against ISCW005600 and AAY66632 antigens affected both cell apoptosis and pathogen infection, and were therefore selected as the candidate tick protective antigens for further characterization (Figures 5A,B).

\section{Characterization of Selected Candidate Tick Protective Antigens}

The first step in the characterization of selected candidate tick protective antigens was the identification of I. ricinus homologs to evaluate their protective potential in both major tick vector species for A. phagocytophilum. The I. ricinus homologs for $I$. scapularis ISCW005600 and AAY66632 antigens corresponded to putative salivary gland secreted proteins lipocalins (Beaufays et al., 2008; Schwarz et al., 2013; Valdés et al., 2016) and a lectin pathway inhibitor (Ribeiro et al., 2006; Schuijt et al., 2011), respectively (Figure 5C). At the amino acid level, over $70 \%$ sequence identity was obtained for both antigens (Figure 5C), suggesting that these proteins are highly conserved in I. scapularis 

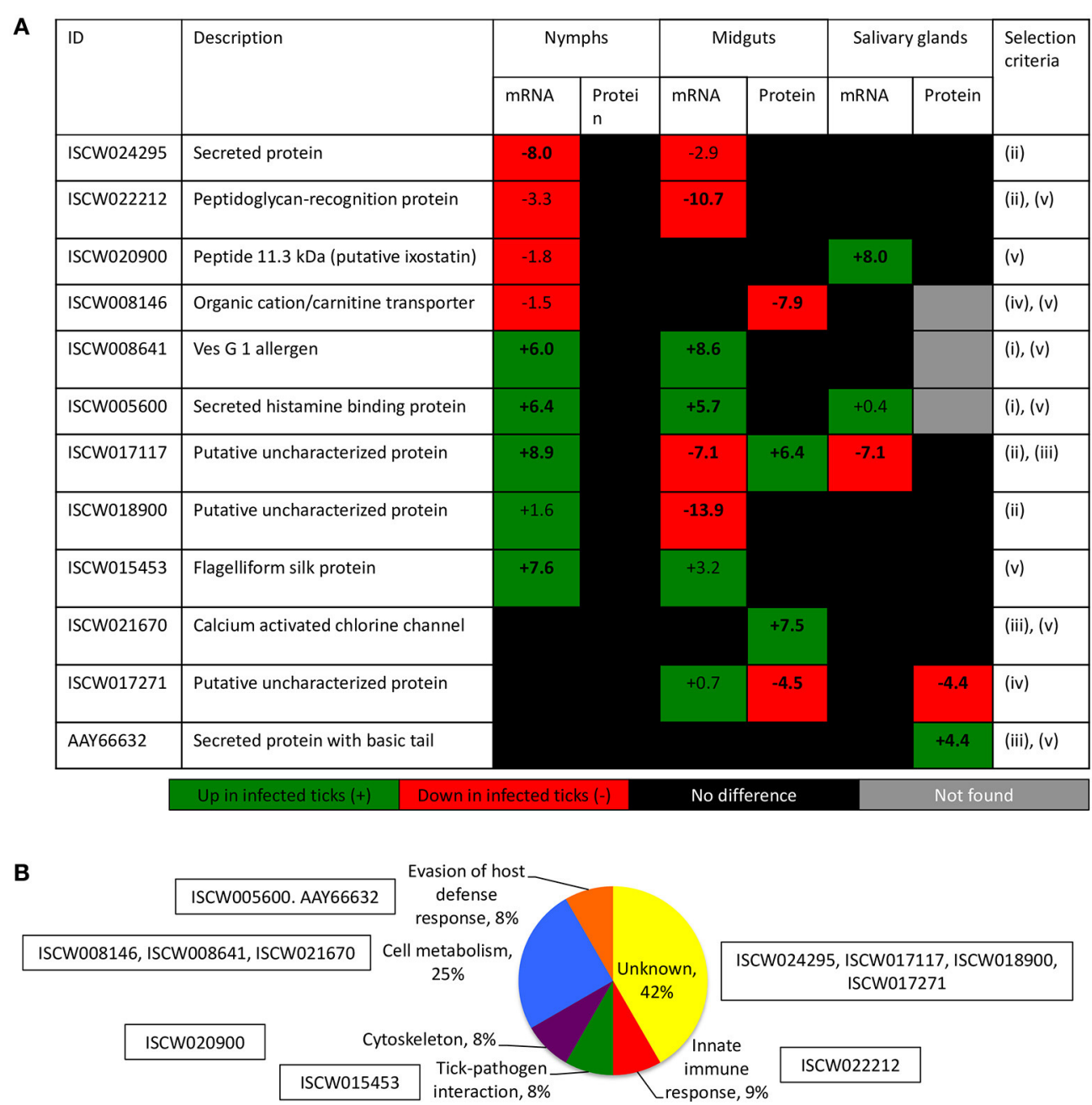

FIGURE 3 | Criteria for the selection of candidate tick protective antigens. (A) Annotation and criteria used for the selection of candidate tick protective antigens. The log2 normalized fold change between infected and uninfected tick samples $(P<0.00003)$ is shown. Bold numbers indicate highly differentially regulated genes (log2 normalized fold change $>5.64$ ) and highly differentially represented proteins (log2 normalized fold change > 3.90) between infected and uninfected tick samples. The selection criteria were (i) highly differentially up-regulated genes in at least two samples, (ii) highly down-regulated genes in at least one sample, (iii) highly differentially over-represented proteins and identified in the I. scapularis proteome, (iv) highly differentially under-represented proteins and identified in the I. scapularis proteome, and/or (v) putative BP in tick-pathogen and tick-host interactions. (B) The GO for BP of selected candidate protective antigens was done with Blast2GO software (version 3.0; http://www.blast2go.com).

and $I$. ricinus, and may be protective in vaccine preparations against both tick vector species.

Experiments were then conducted to characterize the effect of rabbit IgG antibodies against ISCW005600 and AAY66632 antigens in heterologous $I$. ricinus IRE/CTVM20 cells as described before in the homologous I. scapularis ISE6 cells (Figures 5D,E). As in ISE6 tick cells, the results showed that incubation of IRE/CTVM20 tick cells with IgG antibodies against ISCW005600 and AAY66632 antigens affected both cell apoptosis (Figure 5D) and pathogen infection (Figure 5E), supporting the putative effect of vaccination with these antigens in both tick vector species.

Functional analyses were conducted to gain additional insight into the possible protective mechanisms for these antigens. The expression of ISCW005600 and AAY66632 was determined by RT-PCR and did not change in response to A. phagocytophilum infection of ISE6 tick cells (Figure 6A), a result that agreed with previous results of transcriptomics analysis (Villar et al., 2015a; Figure 6B). The IFA in uninfected and A. phagocytophiluminfected $I$. scapularis females showed that as expected, a negative and positive staining was obtained with pre-immune and antiISE6 IgG in infected ticks, respectively (Figures 6Ca-d). The ISCW017271 antigen, which protein levels were highly underrepresented in response to infection in both midguts and salivary glands (Figure 3A), was used to validate proteomics results. The IFA using anti-ISCW017271 IgG antibodies showed a positive staining in uninfected (Figure 6Ce) but not infected cells (Figure 6Cf), thus corroborating the proteomics results. 


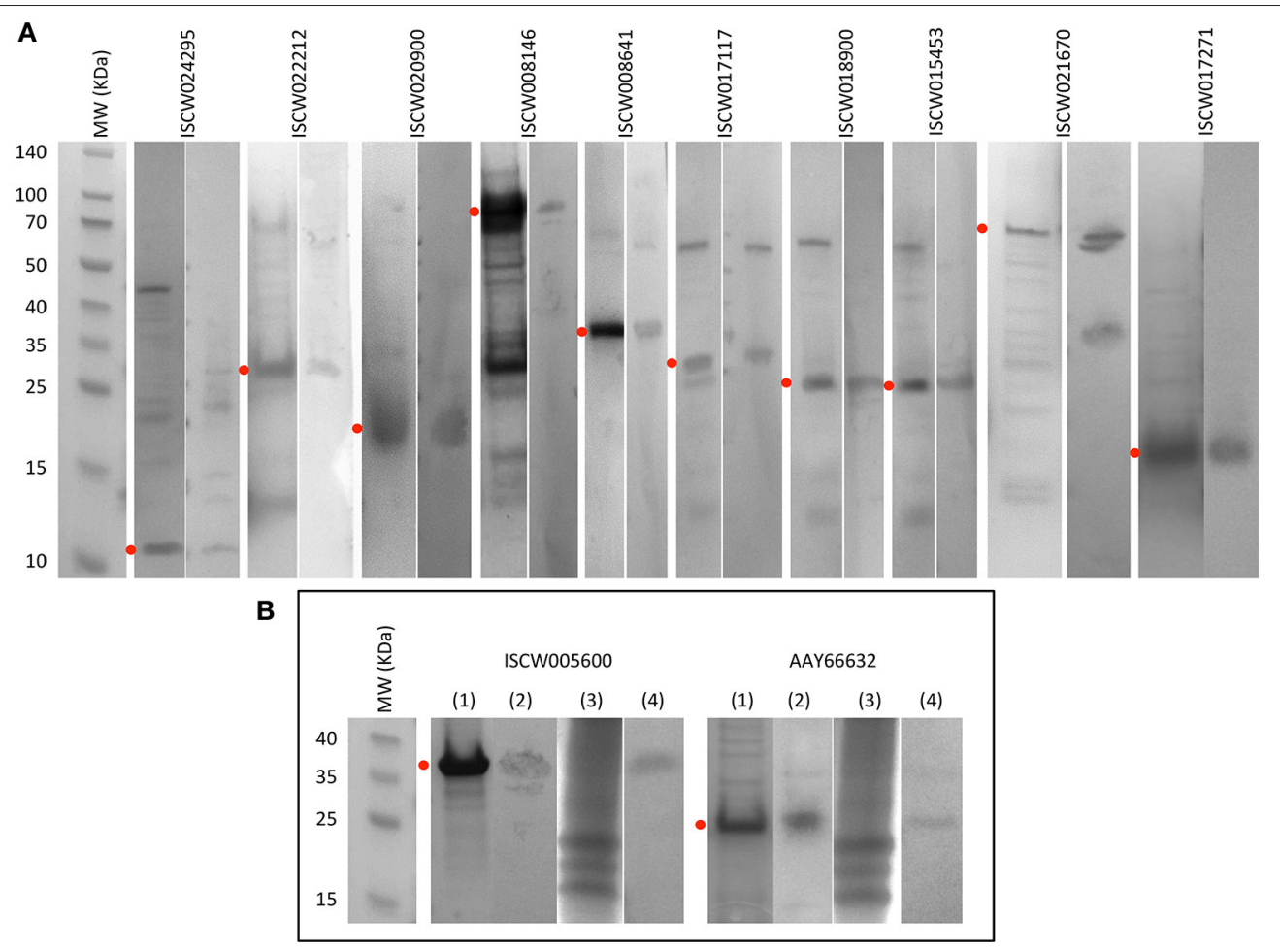

FIGURE 4 | Production of recombinant proteins and rabbit lgG antibodies. (A) SDS-PAGE (left panel) and Western blot analysis (right panel) of selected candidate tick protective recombinant antigens. (B) Antigens selected for further characterization. (1) SDS-PAGE of recombinant antigens. (2) Western blot analysis of recombinant antigens. (3) SDS-PAGE of total proteins from ISE6 tick cells. (4) Western blot analysis of total proteins from ISE6 tick cells. Western blots were performed with IgG antibodies from rabbits immunized with recombinant antigens. Red dots denote the position of the recombinant antigen. Other protein bands in some of the samples correspond to $E$. coli contamination proteins, and aggregation or degradation products of the recombinant antigens.

For the selected candidate tick protective antigens, the IFA with anti-ISCW005600 IgG did not produce any positive staining (Figures $\mathbf{6 C g}-\mathbf{h}$ ), in accordance with proteomics results (Figure 3A). However, for the AAY66632 antigen, a positive staining was obtained in salivary glands from infected ticks after IFA with anti-AAY66632 antibodies (Figures 6Ci,j). The positive staining in infected (Figure 6 $\mathbf{C l}$ ) but not uninfected (Figure 6Ck) ticks formed a membrane-like structure in salivary glands (arrows in Figure $\mathbf{6 C l}$ ), and also corroborated the proteomics results for this antigen (Figure 3A).

Gene knockdown by RNAi in ISE6 tick cells resulted in significantly lower A. phagocytophilum infection levels for both antigens when compared to control cells using the unrelated Rs86 dsRNA (Figure 6D). These results suggested that although ISCW005600 and AAY66632 mRNA levels did not change in response to infection of ISE6 tick cells, which constitute a model for tick hemocytes involved in pathogen infection and immune response (Villar et al., 2015a; Alberdi et al., 2016), they may play a role in $A$. phagocytophilum infection.

These results encouraged a final experiment to evaluate the potential effect of ISCW005600 and AAY66632 as vaccination antigens to reduce tick infestations and reproduction. An artificial tick feeding system using silicone membranes was used in this experiment (Kröber and Guerin, 2007; Krull et al., 2017).
Although the development of standardized in vitro feeding methods for ixodid ticks has been hampered by their complex feeding behavior and the long duration of their blood meal, recent developments provide a valuable tool for the study of tick physiology, tick-host-pathogen interactions and the discovery of drugs and other control interventions without the use of experimental animals (Kröber and Guerin, 2007; Bonnet and Liu, 2012; Sojka et al., 2015; Tajeri et al., 2016; Krull et al., 2017; Trentelman et al., 2017). I. ricinus ticks were selected for artificial feeding and the results shown here supported an effect of antibodies against $I$. scapularis antigens (Figure 4B) on I. ricinus ticks (Figures 5D,E).

On the artificial feeding device, the number of attached ticks was similar between groups, but the number of dead ticks increased after feeding on anti-antigen IgG and was significantly higher in ticks fed on anti-AAY66632 antibodies when compared to control ticks fed on pre-immune IgG (Figure 7). Significant differences were not observed between groups in tick weight, number of ticks with oviposition and egg weight, but a tendency in the reduction in the number of ticks with oviposition was also observed in ticks fed on anti-AAY66632 IgG (Figure 7). Although the number of ticks used for artificial feeding was limited due to experimental conditions, the results suggested an effect of anti-ISCW005600 and anti-AYY66632 antibodies on tick 
A

A

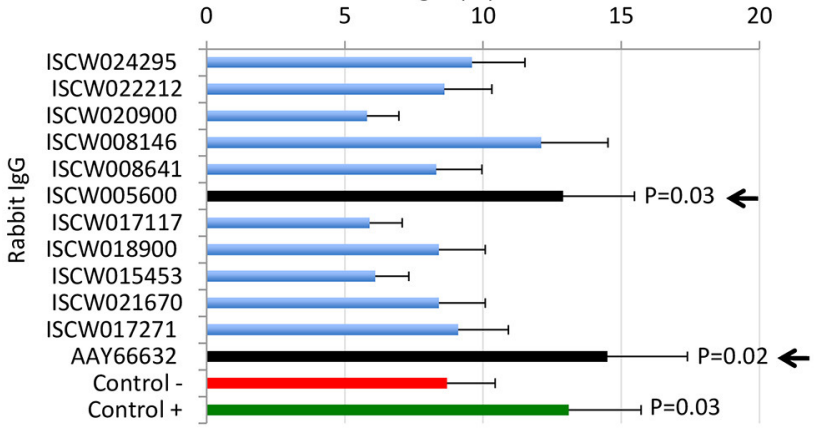

B

A. phagocytophilum normalized DNA levels in ISE6 cells (arbitrary units)

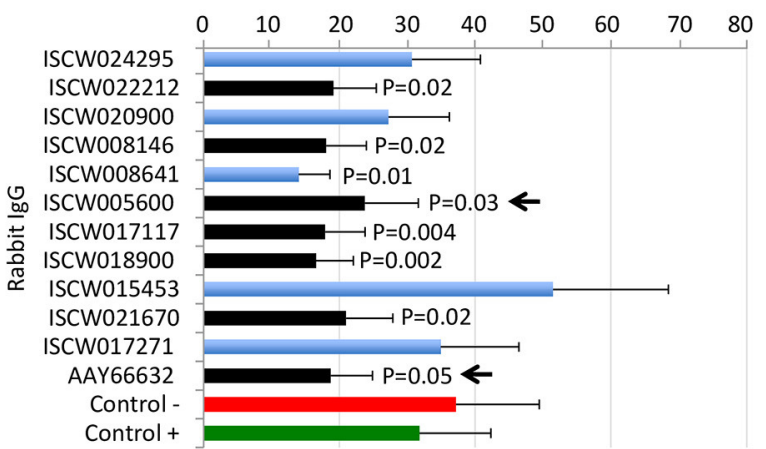

C

\begin{tabular}{|c|c|c|}
\hline I. scapularis & \multicolumn{2}{|c|}{ I. ricinus } \\
\hline & $\begin{array}{l}\text { AM055957 } \\
\text { AM055959 }\end{array}$ & KF451926 \\
\hline ISCW005600 & $\begin{array}{l}\text { nt: } 92 \%, E=0.0 \\
\text { aa: } 86 \%, E=0.0\end{array}$ & \\
\hline AAY 66632 & & $\begin{array}{l}\text { t: } 79 \%, E=2 e-26 \\
\text { a: } 72 \%, E=1 e-41\end{array}$ \\
\hline
\end{tabular}

D

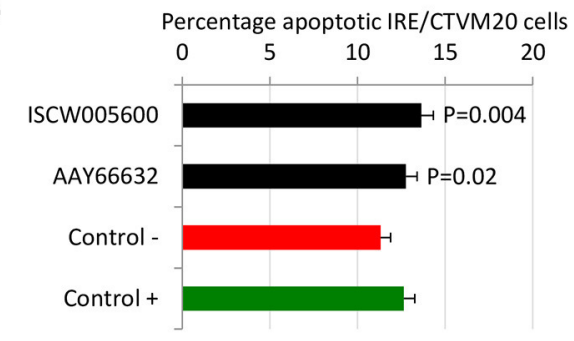

E

A. phagocytophilum normalized DNA levels in IRE/CTVM20 cells (arbitrary units) $\begin{array}{llllll}0 & 10 & 20 & 30 & 40 & 50\end{array}$

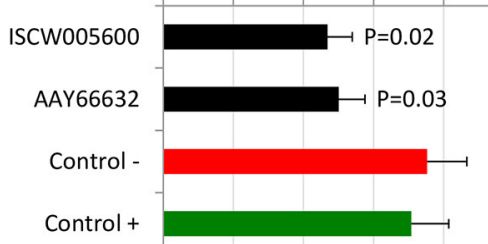

FIGURE 5 | Selection of candidate tick protective antigens. Purified rabbit lgG against recombinant candidate tick protective antigens were incubated with I. scapularis ISE6 and I. ricinus IRE/CTVM20 tick cells before infection with A. phagocytophilum. (A) Characterization of the effect of rabbit IgG antibodies on ISE6 tick cells apoptosis. (B) Characterization of the effect of rabbit IgG antibodies on pathogen infection of ISE6 tick cells. (C) Sequence identity between I. scapularis and I. ricinus homologs. The accession numbers are shown together with corresponding percent identity for nucleotide (nt) and amino acid (aa) sequences and $E$-values. The selected I. scapularis sequences were blasted against the I. ricinus database using the Blastp tool from BLAST, and the sequences with the lowest $E$-value were selected. (D) Characterization of the effect of rabbit IgG antibodies on IRE/CTVM20 tick cells apoptosis. (E) Characterization of the effect of rabbit IgG antibodies on pathogen infection of IRE/CTVM20 tick cells. The percentage of apoptotic cells was determined by flow cytometry after Annexin V-FITC and PI labeling. A. phagocytophilum DNA levels were determined by $m s p 4$ real-time PCR normalizing against tick rpS4. Control cells were incubated with rabbit pre-immune lgG (negative control, Control -) or rabbit anti-ISE6 IgG (positive control, Control +). Results were presented as average + S.D. normalized Ct-values and compared between each treatment and negative control by Student's $t$-test with unequal variance $(P \leq 0.05 ; N=4)$. The selected candidate protective antigens are shown with arrows.

mortality and a reduction in the number of ticks with oviposition for anti-AYY66632 antibodies.

These results suggested that the selected candidate tick protective antigens might constitute effective vaccine antigens to control tick vector infestations and prevent or control pathogen infection, and therefore could be selected for future vaccination trials.

\section{Putative Mechanisms of Protection for Vaccines Based on Selected Candidate Tick Protective Antigens}

After the successful completion of the main objective of this study, which was the identification of tick candidate tick protective antigens for the control of vector infestations and $A$. phagocytophilum infection, a question arose about the putative protective mechanisms of the selected candidate protective antigens. The answer to this question may assist in the selection of additional candidate protective antigens following the vaccinomics pipeline (Figure 1), and the evaluation of possible combinations of antigens with different functions to enhance vaccine efficacy (de la Fuente and Merino, 2013).

Both selected candidate tick protective antigens were grouped into the evasion of host defense response BP (Figure 3B). The ISCW005600 secreted histamine binding protein appears to be a salivary lipocalin (Beaufays et al., 2008; Schwarz et al., 2013). Lipocalins are a family of salivary gland secreted proteins that play a role in evasion of host immune and inflammatory responses by competing for histamine or serotonin binding (Paesen et al., 2000; Mans, 2005; Beaufays et al., 2008; Valdés, 2014; Valdés et al., 2016). Therefore, these proteins play an important role during tick feeding. The genes encoding for these proteins are up-regulated during tick feeding (Kim et al., 2016; Valdés et al., 2016; Ribeiro et al., 2017) and pathogen infection (Ayllón et al., 2015; Valdés et al., 2016). Additionally, lipocalins were also produced in tick midguts and up-regulated 


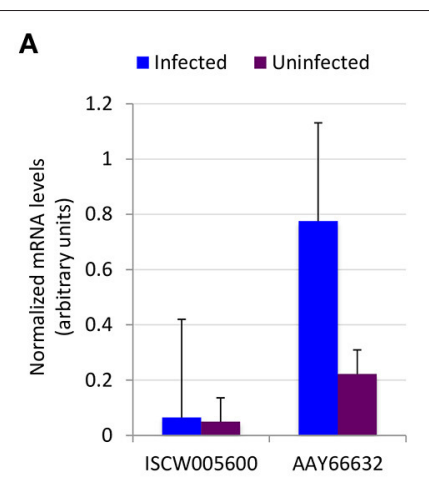

B

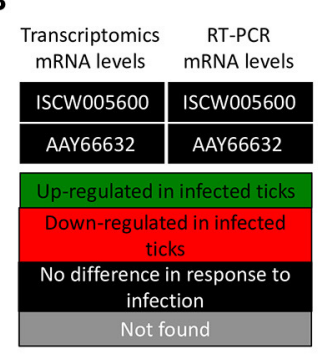

D

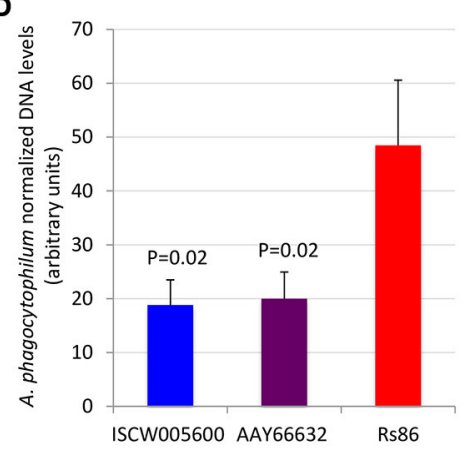

C

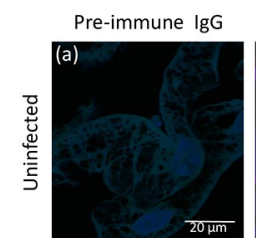

Anti-ISE6 IgG
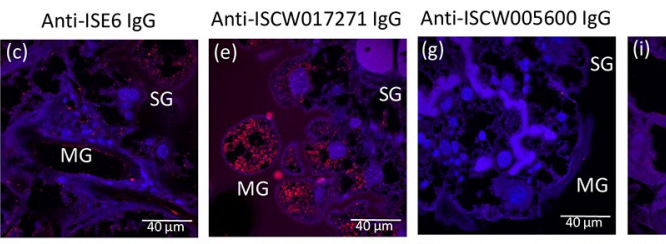

Anti-AAY66632 IgG
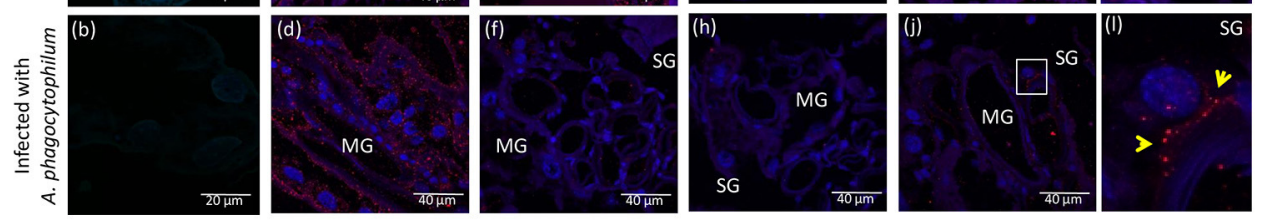

FIGURE 6 | Functional characterization of selected candidate tick protective antigens. (A) Results of the RT-PCR analysis of the expression of ISCW005600 and AAY66632 genes in uninfected and A. phagocytophilum-infected ISE6 tick cells. Results were presented as average + S.D. normalized Ct-values and compared between infected and uninfected cells by Student's $t$-test with unequal variance $(P \leq 0.05 ; N=4)$. (B) Comparison of the transcriptomics and RT-PCR results for mRNA levels of ISCW005600 and AAY66632 genes in ISE6 tick cells in response to A. phagocytophilum infection. Transcriptomics results were obtained from Villar et al. (2015a). (C) Representative images of imunofluorescence analysis of uninfected (a,c,e,g,i,k) and A. phagocytophilum-infected (b,d,f,h,j, ,l) adult female I. scapularis midguts (MG) and salivary glands (SG). Tick tissues were stained with rabbit pre-immune control lgG (a,b), anti-ISE6 tick cells IgG (c,d), or anti-tick antigens IgG (e-l) labeled with RFP (red) and DAPI (blue). Yellow arrows illustrate a positive staining for AAY66632 in the SG sections in white squares in infected (I) but not uninfected (k) ticks. (D) The A. phagocytophilum DNA levels were determined after RNAi in infected ISE6 tick cells treated with ISCW005600 and AAY66632 dsRNAs or control Rs 86 dsRNA. A. phagocytophilum DNA levels were determined by msp4 real-time PCR normalizing against tick rpS4. Results are shown as average + S.D. normalized Ct-values and compared between treated and control groups by Student's $t$-test with unequal variance $(P<0.05$; $N=5$ biological replicates).

in response to A. phagocytophilum infection (Ayllón et al., 2015; Figure 3A), suggesting as reported in other organisms (Cassidy and Martineau, 2014; Abella et al., 2015) a role for these proteins in tick innate immune response to infection. Therefore, lipocalins may have a dual role in tick-pathogen interactions. These proteins may facilitate pathogen transmission by reducing host inflammatory responses (Valdés et al., 2016), but control tick infection by depleting strategic compounds for pathogens (Ferreira et al., 2015). In humans, lipocalins have also been shown to regulate apoptosis by inducing or inhibiting this process under different physiological conditions (Chakraborty et al., 2012; Abella et al., 2015). Based on the results obtained here with antiISCW005600 antibodies and RNAi (Figures 5A,B,D,E, 6D, 7), ISCW005600 may function to inhibit tick cell apoptosis and facilitate $A$. phagocytophilum infection with a possible role during tick feeding (Figure 7). Therefore, the proposed protective mechanisms for vaccines containing this antigen may include reduction of tick infestations by increasing cell apoptosis and reducing protective capacity to host response while reducing pathogen infection and transmission. Tick lipocalins have been proposed before as vaccine antigens for the control of tick infestations (de Castro et al., 2016; Manzano-Román et al., 2016), but only low partial protection have been reported in soft ticks, Ornithodoros moubata fed on immunized rabbits (ManzanoRomán et al., 2016).

The AAY66632 antigen is a secreted lectin pathway inhibitor (Ribeiro et al., 2006; Schuijt et al., 2011), which is involved in the inhibition of the innate immune response complement lectin pathway (CLP). The CLP is involved in host response to infection with different pathogens (Evans-Osses et al., 2013). The CLP is activated when mannan-binding lectins or ficolins bind to patterns of carbohydrates or acetyl groups on the surface of protozoan, virus, fungi, or bacteria (Runza et al., 2008; Héja et al., 2012; Evans-Osses et al., 2013). In ticks, the inhibition of the complement system during and after blood feeding is critical for tick feeding success and development by minimizing damage to the intestinal epithelium as well as avoiding inflammation and opsonization of salivary molecules at the bite site (Wikel and Allen, 1977; Franco et al., 2016). Therefore, complement inhibitors are present in both tick saliva and midgut (Barros et al., 


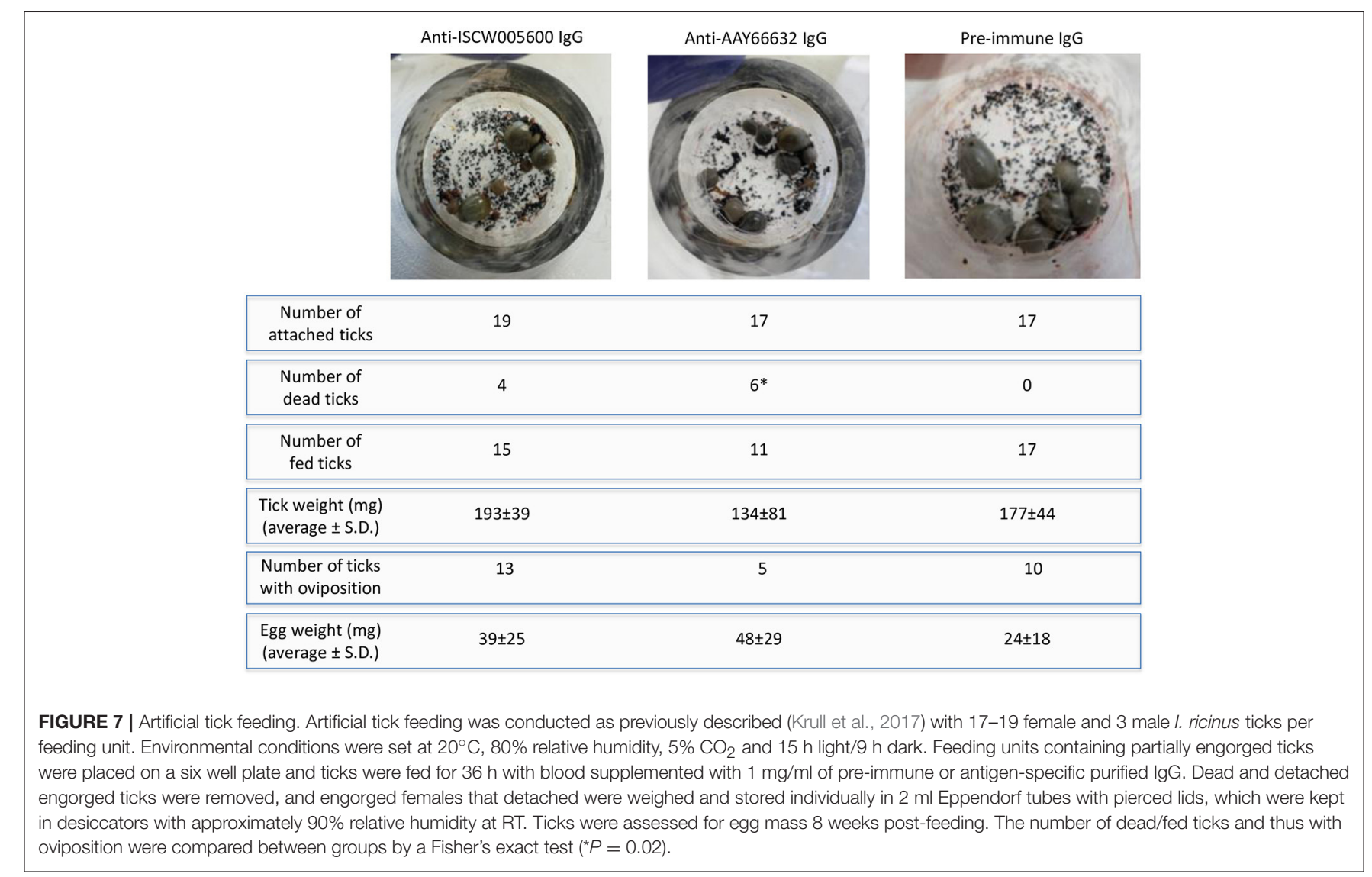

2009; Mendes-Sousa et al., 2013; Ayllón et al., 2015) (Figure 3A). The presence and activity of salivary anti-complement molecules has been well characterized in Ixodes spp. ticks including the A. phagocytophilum vectors, I. scapularis (Valenzuela et al., 2000; Tyson et al., 2007, 2008, Schuijt et al., 2011) and I. ricinus (Lawrie et al., 1999, 2005, Daix et al., 2007; Couvreur et al., 2008). Moreover, tick lectin pathway inhibitors have been shown to facilitate Borrelia burgdorferi pathogen infection and transmission (Schuijt et al., 2011; Wagemakers et al., 2016). Our results supported a role for AAY66632 in tick feeding success (Figure 7), the inhibition of tick cell apoptosis (Figures 5A,D) and facilitation of $A$. phagocytophilum infection (Figures 5B,E, 6D). Therefore, the proposed protective mechanisms for vaccines based on this antigen may include reduction of tick infestations by affecting tick attachment and/or feeding, while reducing pathogen infection and transmission. The protective capacity of vaccines containing this antigen has not been reported.

\section{CONCLUSIONS}

The main objective of this study was to apply a vaccinomics approach to the identification and characterization of candidate tick protective antigens for the control of vector infestations and A. phagocytophilum infection. The vaccinomics pipeline developed in this study was applied to tick- $A$. phagocytophilum interactions and resulted in the identification of two candidate tick protective antigens that could be selected for future vaccination trials. The results showed that $I$. scapularis ISCW005600 and AAY66632 and I. ricinus homologs constitute candidate protective antigens for the control of vector infestations and A. phagocytophilum infection. Both lipocalin (ISCW005600) and lectin pathway inhibitor (AAY66632) are involved in the tick evasion of host defense response and pathogen infection and transmission, but targeting different immune response pathways. Therefore, based on the putative function of these antigens, vaccine protective mechanisms were proposed that supported antigen combination to improve vaccine efficacy. The vaccinomics pipeline proposed here could be used to continue the identification and characterization of candidate tick protective antigens for the development of effective vaccines for the prevention and control of HGA, TBF, and other forms of anaplasmosis caused by A. phagocytophilum.

\section{AUTHOR CONTRIBUTIONS}

JD conceived the study. MC, PA, IF, MV, CK, and AN performed the experiments. MC, PA, MV, CK, AN, and JD performed data analyses. JD, MC, and PA wrote the paper, and 
other coauthors made additional suggestions and approved the manuscript.

\section{FUNDING}

This research was partially supported by the Ministerio de Economia, Industria y Competitividad (Spain) grant BFU201679892-P, and the CSIC grant 201440E098 to JD. The University of Castilla-La Mancha (UCLM), Spain, supported the stay of MC with AN's group at the Free University of Berlin (Germany). MV and IF were supported by the Research Plan of the UCLM, Spain.

\section{REFERENCES}

Abella, V., Scotece, M., Conde, J., Gómez, R., Lois, A., Pino, J., et al. (2015). The potential of lipocalin-2/NGAL as biomarker for inflammatory and metabolic diseases. Biomarkers 20, 565-571. doi: 10.3109/1354750X.2015.1123354

Alberdi, P., Ayllón, N., Cabezas-Cruz, A., Bell-Sakyi, L., Zweygarth, E., Stuen, S., et al. (2015). Infection of Ixodes spp. tick cells with different Anaplasma phagocytophilum isolates induces the inhibition of apoptotic cell death. Ticks Tick Borne Dis. 6, 758-767. doi: 10.1016/j.ttbdis.2015.07.001

Alberdi, P., Mansfield, K. L., Manzano-Román, R., Cook, C., Ayllón, N., Villar, M., et al. (2016). Tissue-specific signatures in the transcriptional response to Anaplasma phagocytophilum infection of Ixodes scapularis and Ixodes ricinus tick cell lines. Front. Cell. Infect. Microbiol. 6:20. doi: 10.3389/fcimb.2016.00020

Altschul, S. F., Gish, W., Miller, W., Myers, E. W., and Lipman, D. J. (1990). Basic local alignment search tool. J. Mol. Biol. 215, 403-410. doi: 10.1016/S0022-2836(05)80360-2

Antunes, S., Merino, O., Mosqueda, J., Moreno-Cid, J. A., Bell-Sakyi, L., Fragkoudis, R., et al. (2014). Tick capillary feeding for the study of proteins involved in tick-pathogen interactions as potential antigens for the control of tick infestation and pathogen infection. Parasit. Vectors 7:42. doi: 10.1186/1756-3305-7-42

Asanovich, K. M., Bakken, J. S., Madigan, J. E., Aguero-Rosenfeld, M., Wormser, G. P., and Dumler, J. S. (1997). Antigenic diversity of granulocytic Ehrlichia isolates from humans in Wisconsin and New York and a horse in California. J. Infect. Dis. 176, 1029-1034. doi: 10.1086/516529

Ayllón, N., Villar, M., Busby, A. T., Kocan, K. M., Blouin, E. F., BonzónKulichenko, E., et al. (2013). Anaplasma phagocytophilum inhibits apoptosis and promotes cytoskeleton rearrangement for infection of tick cells. Infect. Immun. 81, 2415-2425. doi: 10.1128/IAI.00194-13

Ayllón, N., Villar, M., Galindo, R. C., Kocan, K. M., Šíma, R., López, J. A., et al. (2015). Systems biology of tissue-specific response to Anaplasma phagocytophilum reveals differentiated apoptosis in the tick vector Ixodes scapularis. PLoS Genet. 11:e1005120. doi: 10.1371/journal.pgen.1005120

Bakken, J. S., and Dumler, J. S. (2015). Human granulocytic anaplasmosis. Infect. Dis. Clin. North Am. 29, 341-355. doi: 10.1016/j.idc.2015.02.007

Barros, V. C., Assumpcao, J. G., Cadete, A. M., Santos, V. C., Cavalcante, R. R., Araujo, R. N., et al. (2009). The role of salivary and intestinal complement system inhibitors in the midgut protection of triatomines and mosquitoes. PLoS ONE 4:e6047. doi: 10.1371/journal.pone.0006047

Beaufays, J., Adam, B., Decrem, Y., Prevot, P. P., Santini, S., Brasseur, R., et al. (2008). Ixodes ricinus tick lipocalins: identification, cloning, phylogenetic analysis and biochemical characterization. PLoS ONE 3:e3941. doi: 10.1371/journal.pone.0003941

Bell-Sakyi, L., Zweygarth, E., Blouin, E. F., Gould, E. A., and Jongejan, F. (2007). Tick cell lines: tools for tick and tick-borne disease research. Trends Parasitol. 23, 450-457. doi: 10.1016/j.pt.2007.07.009

Bonnet, S., and Liu, X. Y. (2012). Laboratory artificial infection of hard ticks: a tool for the analysis of tick-borne pathogen transmission. Acarologia. 52, 453-464. doi: 10.1051/acarologia/20122068

Cabezas-Cruz, A., Alberdi, P., Ayllón, N., Valdés, J. J., Pierce, R., Villar, M., et al. (2016). Anaplasma phagocytophilum increases the levels of histone modifying enzymes to inhibit cell apoptosis and facilitate pathogen
The funders had no role in study design, data collection and interpretation, or the decision to submit the work for publication.

\section{ACKNOWLEDGMENTS}

We thank Ulrike Munderloh (University of Minnesota, USA) and Lesley Bell-Sakyi (the Tick Cell Biobank, The Pirbright Institute; now at the Institute of Infection and Global Health, University of Liverpool, UK) for providing ISE6 and IRE/CTVM20 cell lines, respectively. Katherine M. Kocan (Oklahoma State University, USA) is acknowledged for providing tick photographs.

infection in the tick vector Ixodes scapularis. Epigenetics 11, 303-319. doi: 10.1080/15592294.2016.1163460

Cabezas-Cruz, A., Alberdi, P., Valdés, J. J., Villar, M., and de la Fuente, J. (2017a). Anaplasma phagocytophilum infection subverts carbohydrate metabolic pathways in the tick vector, Ixodes scapularis. Front. Cell. Infect. Microbiol. 7:23. doi: 10.3389/fcimb.2017.00023

Cabezas-Cruz, A., Alberdi, P., Valdés, J. J., Villar, M., and de la Fuente, J. (2017b). Remodeling of tick cytoskeleton in response to infection with Anaplasma phagocytophilum. Front. Biosci. 22, 1830-1844.

Cassidy, J. P., and Martineau, A. R. (2014). Innate resistance to tuberculosis in man, cattle and laboratory animal models: nipping disease in the bud? J. Comp. Pathol. 151, 291-308. doi: 10.1016/j.jcpa.2014.08.001

Chakraborty, S., Kaur, S., Guha, S., and Batra, S. K. (2012). The multifaceted roles of neutrophil gelatinase associated lipocalin (NGAL) in inflammation and cancer. Biochim. Biophys. Acta 1826, 129-169. doi: 10.1016/j.bbcan.2012.03.008

Chmelař, J., Kotál, J., Karim, S., Kopacek, P., Francischetti, I. M., Pedra, J. H., et al. (2016). Sialomes and mialomes: a systems-biology view of tick tissues and tickhost interactions. Trends Parasitol. 32, 242-254. doi: 10.1016/j.pt.2015.10.002

Contreras, M., Alberdi, P., Mateos-Hernández, L., Fernández de Mera, I. G., García-Pérez, A. L., et al. (2017). Anaplasma phagocytophilum MSP4 and HSP70 proteins are involved in interactions with host cells during pathogen infection. Front. Cell. Infect. Microbiol. 7:307. doi: 10.3389/fcimb.2017. 00307

Contreras, M., Villar, M., Alberdi, P., and de la Fuente, J. (2016). Vaccinomics approach to tick vaccine development. Methods Mol. Biol. 1404, 275-286. doi: 10.1007/978-1-4939-3389-1_19

Couvreur, B., Beaufays, J., Charon, C., Lahaye, K., Gensale, F., Denis, V., et al. (2008). Variability and action mechanism of a family of anticomplement proteins in Ixodes ricinus. PLoS ONE 3:e1400. doi: 10.1371/journal.pone.0001400

Cramaro, W. J., Revets, D., Hunewald, O. E., Sinner, R., Reye, A. L., and Muller, C. P. (2015). Integration of Ixodes ricinus genome sequencing with transcriptome and proteome annotation of the naïve midgut. BMC Genomics 16:871. doi: 10.1186/s12864-015-1981-7

Daix, V., Schroeder, H., Praet, N., Georgin, J. P., Chiappino, I., Gillet, L., et al. (2007). Ixodes ticks belonging to the Ixodes ricinus complex encode a family of anticomplement proteins. Insect Mol. Biol. 16, 155-166. doi: 10.1111/j.1365-2583.2006.00710.x

de Castro, M. H., de Klerk, D., Pienaar, R., Latif, A. A., Rees, D. J., and Mans, B. J. (2016). De novo assembly and annotation of the salivary gland transcriptome of Rhipicephalus appendiculatus male and female ticks during blood feeding. Ticks Tick Borne Dis. 7, 536-548. doi: 10.1016/j.ttbdis.2016.01.014

de la Fuente, J., Antunes, S., Bonnet, S., Cabezas-Cruz, A., Domingos, A., EstradaPeña, A., et al. (2017). Tick-pathogen interactions and vector competence: identification of molecular drivers for tick-borne diseases. Front. Cell. Infect. Microbiol. 7:114. doi: 10.3389/fcimb.2017.00114

de la Fuente, J., and Contreras, M. (2015). Tick vaccines: current status and future directions. Expert Rev. Vaccines. 14, 1367-1376. doi: 10.1586/14760584.2015.1076339

de la Fuente, J., Kopáček, P., Lew-Tabor, A., and Maritz-Olivier, C. (2016a). Strategies for new and improved vaccines against ticks and tick-borne diseases. Parasite Immunol. 38, 754-769. doi: 10.1111/pim.12339 
de la Fuente, J., Waterhouse, R. M., Sonenshine, D. E., Roe, R. M., Ribeiro, J. M., et al. (2016b). Tick genome assembled: new opportunities for research on tick-host-pathogen interactions. Front. Cell. Infect. Microbiol. 6:103. doi: 10.3389/fcimb.2016.00103

de la Fuente, J., Estrada-Peña, A., Cabezas-Cruz, A., and Kocan, K. M. (2016c). Anaplasma phagocytophilum uses common strategies for infection of ticks and vertebrate hosts. Trends. Microbiol. 24, 173-180. doi: 10.1016/j.tim.2015.12.001

de la Fuente, J., Villar, M., Cabezas-Cruz, A., Estrada-Peña, A., Ayllón, N., and Alberdi, P. (2016d). Tick-host-pathogen interactions: conflict and cooperation. PLoS. Pathog. 12:e1005488. doi: 10.1371/journal.ppat.1005488

de la Fuente, J., and Merino, O. (2013). Vaccinomics, the new road to tick vaccines. Vaccine 31, 5923-5929. doi: 10.1016/j.vaccine.2013.10.049

Dugat, T., Lagrée, A. C., Maillard, R., Boulouis, H. J., and Haddad, N. (2015). Opening the black box of Anaplasma phagocytophilum diversity: current situation and future perspectives. Front. Cell. Infect. Microbiol. 5:61. doi: 10.3389/fcimb.2015.00061

Dumler, J. S., Barbet, A. C., Bekker, C. P. J., Dasch, G. A., Palmer, G. H., Ray, S. C., et al. (2001). Reorganization of the genera in the families Rickettsiaceae and Anaplasmataceae in the order Rickettsiales: unification of some species of Ehrlichia with Anaplasma, Cowdria with Ehrlichia and Ehrlichia with Neorickettsia, descriptions subjective synonyms of Ehrlichia phagocytophila. Int. J. Syst. Evol. Microbiol. 51, 2145-2165. doi: 10.1099/00207713-51-6-2145

Evans-Osses, I., de Messias-Reason, I., and Ramirez, M. I. (2013). The emerging role of complement lectin pathway in trypanosomatids: molecular bases in activation, genetic deficiencies, susceptibility to infection, and complement system-based therapeutics. Sci. World J. 2013:675898. doi: $10.1155 / 2013 / 675898$

Ferreira, A. C., Dá Mesquita, S., Sousa, J. C., Correia-Neves, M., Sousa, N., Palha, J. A. et al. (2015). From the periphery to the brain: Lipocalin-2, a friend or foe? Prog Neurobiol. 131, 120-136. doi: 10.1016/j.pneurobio.2015.06.005

Foggie, A. (1951). Studies on the infectious agent of tick-borne fever in sheep. J. Path. Bact. 63, 1-15. doi: 10.1002/path.1700630103

Franco, P. F., Silva, N. C., Fazito do Vale, V., Abreu, J. F., Santos, V. C., Gontijo, N. F., et al. (2016). Inhibition of the classical pathway of the complement system by saliva of Amblyomma cajennense (Acari: Ixodidae). Exp. Parasitol. 164, 91-96. doi: 10.1016/j.exppara.2016.03.002

Genomic Resources Development Consortium, Contreras, M., de la Fuente, J., Estrada-Peña, A., Grubhoffer, L., and Tobes, R. (2014). Transcriptome sequence divergence between Lyme disease tick vectors, Ixodes scapularis and Ixodes ricinus. Genomic Resources Notes. Mol. Ecol. Resour. 14, 1095. doi: 10.1111/1755-0998.12298

Gordon, W. S., Brownlee, A., Wilson, D. R., and MacLeod, J. (1932). Tick-borne fever. J. Comp. Path. 45, 301-302. doi: 10.1016/S0368-1742(32)80025-1

Gulia-Nuss, M., Nuss, A. B., Meyer, J. M., Sonenshine, D. E., Roe, R. M., Waterhouse, R. M., et al. (2016). Genomic insights into the Ixodes scapularis tick vector of Lyme disease. Nat. Commun. 7:10507. doi: $10.1038 /$ ncomms 10507

Héja, D., Kocsis, A., Dobó, J., Szilágyi, K., Szász, R., Závodszky, P., et al. (2012). Revised mechanism of complement lectin-pathway activation revealing the role of serine protease MASP-1 as the exclusive activator of MASP-2. Proc. Natl. Acad. Sci. U.S.A. 109, 10498-10503. doi: 10.1073/pnas.1202588109

Kim, T. K., Tirloni, L., Pinto, A. F., Moresco, J., Yates, J. R. III., da Silva Vaz, I. Jr., et al. (2016). Ixodes scapularis tick saliva proteins sequentially secreted every $24 \mathrm{~h}$ during blood feeding. PLoS Negl. Trop. Dis. 10:e0004323. doi: 10.1371/journal.pntd.0004323

Kocan, K. M., Busby, A. T., Allison, R. W., Breshears, M. A., Coburn, L., Galindo, R. C., et al. (2012). Sheep experimentally-infected with a human isolate of Anaplasma phagocytophilum serve as a host for infection of Ixodes scapularis. Ticks Tick Borne Dis. 3, 147-153. doi: 10.1016/j.ttbdis.2012.01.004

Kotsyfakis, M., Schwarz, A., Erhart, J., and Ribeiro, J. M. (2015). Tissue- and timedependent transcription in Ixodes ricinus salivary glands and midguts when blood feeding on the vertebrate host. Sci. Rep. 5:9103. doi: 10.1038/srep09103

Kröber, T., and Guerin, P. M. (2007). An in vitro feeding assay to test acaricides for control of hard ticks. Pest Manag. Sci. 63, 17-22. doi: 10.1002/ps. 1293

Krull, C., Böhme, B., Clausen, P. H., and Nijhof, A. M. (2017). Optimization of an artificial tick feeding assay for Dermacentor reticulatus. Parasit. Vectors 10:60. doi: $10.1186 /$ s13071-017-2000-4
Kurtti, T. J., Munderloh, U. G., Andreadis, T. G., Magnarelli, L. A., and Mather, T. N. (1996). Tick cell culture isolation of an intracellular prokaryote from the tick Ixodes scapularis. J. Inv. Path. 67, 318-321. doi: 10.1006/jipa.1996.0050

Lawrie, C. H., Randolph, S. E., and Nuttall, P. A. (1999). Ixodes ticks: serum species sensitivity of anticomplement activity. Exp. Parasitol. 93, 207-214. doi: 10.1006/expr.1999.4456

Lawrie, C. H., Sim, R. B., and Nuttall, P. A. (2005). Investigation of the mechanisms of anti-complement activity in Ixodes ricinus ticks. Mol. Immunol. 42, 31-38. doi: 10.1016/j.molimm.2004.07.001

Madden, T. L., Tatusov, R. L., and Zhang, J. (1996). Applications of network BLAST server. Meth. Enzymol. 266, 131-141. doi: 10.1016/S0076-6879(96)66011-X

Mans, B. J. (2005). Tick histamine-binding proteins and related lipocalins: potential as therapeutic agents. Curr. Opin. Investig. Drugs. 6, 1131-1135.

Manzano-Román, R., Díaz-Martín, V., Oleaga, A., Obolo-Mvoulouga, P., and Pérez-Sánchez, R. (2016). TSGP4 from Ornithodoros moubata: molecular cloning, phylogenetic analysis and vaccine efficacy of a new member of the lipocalin clade of cysteinyl leukotriene scavengers. Vet. Parasitol. 227, 130-137. doi: 10.1016/j.vetpar.2016.08.005

Mendes-Sousa, A. F., Nascimento, A. A. S., Queiroz, D. C., Vale, V. F., Fujiwara, R. T., Araújo, R. N., et al. (2013). Different host complement systems and their interactions with saliva from Lutzomyia longipalpis (Diptera, Psychodidae) and Leishmania infantum promastigotes. PLoS ONE 8:e79787. doi: 10.1371/journal.pone.0079787

Merino, M., Antunes, S., Mosqueda, J., Moreno-Cid, J. A., Pérez de la Lastra, J. M., Rosario-Cruz, R., et al. (2013). Vaccination with proteins involved in tick-pathogen interactions reduces vector infestations and pathogen infection. Vaccine 31, 5889-5896. doi: 10.1016/j.vaccine.2013.09.037

Moreno-Cid, J. A., Pérez de la Lastra, J. M., Villar, M., Jiménez, M., Pinal, R., Estrada-Peña, A., et al. (2013). Control of multiple arthropod vector infestations with subolesin/akirin vaccines. Vaccine 31, 1187-1196. doi: 10.1016/j.vaccine.2012.12.073

Munderloh, U. G., Jauron, S. D., Fingerle, V., Leitritz, L., Hayes, S. F., Hautman, J. M., et al. (1999). Invasion and intracellular development of the human granulocytic ehrlichiosis agent in tick cell culture. J. Clin. Microbiol. 37, 2518-2524.

Paesen, G. C., Adams, P. L., Nuttall, P. A., and Stuart, D. L. (2000). Tick histaminebinding proteins: lipocalins with a second binding cavity. Biochim. Biophys. Acta 1482, 92-101. doi: 10.1016/S0167-4838(00)00168-0

Ribeiro, J. M., Alarcon-Chaidez, F., Francischetti, I. M., Mans, B. J., Mather, T. N., Valenzuela, J. G., et al. (2006). An annotated catalog of salivary gland transcripts from Ixodes scapularis ticks. Insect Biochem. Mol. Biol. 36, 111-129. doi: 10.1016/j.ibmb.2005.11.005

Ribeiro, J. M., Slovák, M., and Francischetti, I. M. (2017). An insight into the sialome of Hyalomma excavatum. Ticks Tick Borne Dis. 8, 201-207. doi: 10.1016/j.ttbdis.2016.08.011

Runza, V. L., Schwaeble, W., and Männel, D. N. (2008). Ficolins: novel pattern recognition molecules of the innate immune response. Immunobiology 213, 297-306. doi: 10.1016/j.imbio.2007.10.009

Schuijt, T. J., Coumou, J., Narasimhan, S., Dai, J., Deponte, K., Wouters, D., et al. (2011). A tick mannose-binding lectin inhibitor interferes with the vertebrate complement cascade to enhance transmission of the Lyme disease agent. Cell Host Microbe 10, 136-146. doi: 10.1016/j.chom.2011.06.010

Schwarz, A., Tenzer, S., Hackenberg, M., Erhart, J., Gerhold-Ay, A., Mazur, J., et al. (2014). A systems level analysis reveals transcriptomic and proteomic complexity in Ixodes ricinus midgut and salivary glands during early attachment and feeding. Mol. Cell. Proteomics 13, 2725-2735. doi: 10.1074/mcp.M114.039289

Schwarz, A., von Reumont, B. M., Erhart, J., Chagas, A. C., Ribeiro, J. M., and Kotsyfakis, M. (2013). De novo Ixodes ricinus salivary gland transcriptome analysis using two next-generation sequencing methodologies. FASEB J. 27, 4745-4756. doi: 10.1096/fj.13-232140

Severo, M. S., Pedra, J. H. F., Ayllón, N., Kocan, K. M., and de la Fuente, J. (2015). "Anaplasma," in Molecular Medical Microbiology, 2nd Edn, eds T. YiWei, L. Dongyou, P. R. Ian, J. D. Schwartzman, and M. Sussman (London: Academic Press; Elsevier), 2033-2042.

Shaw, D. K., Wang, X., Brown, L. J., Oliva Chávez, A. S., Reif, K. E., Smith, A. A., et al. (2017). Infection-derived lipids elicit an immune deficiency circuit in arthropods. Nat. Commun. 8:14401. doi: 10.1038/ncomms14401 
Sojka, D., Pytelkova, J., Perner, J., Horn, M., Konvickova, J., Schrenkova, J., et al. (2015). Multienzyme degradation of host serum albumin in ticks. Ticks Tick Borne Dis. 7, 604-613. doi: 10.1016/j.ttbdis.2015.12.014

Stuen, S., Granquist, E. G., and Silaghi, C. (2013). Anaplasma phagocytophiluma widespread multi-host pathogen with highly adaptive strategies. Front. Cell. Infect. Microbiol. 3:31. doi: 10.3389/fcimb.2013.00031

Stuen, S., Okstad, W., Artursson, K., Al-Khedery, B., Barbet, A., and Granquist, E. G. (2015). Lambs immunized with an inactivated variant of Anaplasma phagocytophilum. Acta Vet. Scand. 57:40. doi: 10.1186/s13028-015-0131-1

Tajeri, S., Razmi, G., and Haghparast, A. (2016). Establishment of an artificial tick feeding system to study Theileria lestoquardi infection. PLOS ONE 11:e0169053. doi: 10.1371/journal.pone.0169053

Thomas, V., and Fikrig, E. (2007). Anaplasma phagocytophilum specifically induces tyrosine phosphorylation of ROCK1 during infection. Cell. Microbiol. 9, 1730-1737. doi: 10.1111/j.1462-5822.2007.00908.x

Trentelman, J. J., Kleuskens, J. A., van de Crommert, J., and Schetters, T. P. (2017). A new method for in vitro feeding of Rhipicephalus australis (formerly Rhipicephalus microplus) larvae: a valuable tool for tick vaccine development. Parasit. Vectors 10:153. doi: 10.1186/s13071-017-2081-0

Tyson, K., Elkins, C., Patterson, H., Fikrig, E., and De Silva, A. (2007). Biochemical and functional characterization of Salp20, an Ixodes scapularis tick salivary protein that inhibits the complement pathway. Insect Mol. Biol. 16, 469-479. doi: 10.1111/j.1365-2583.2007.00742.x

Tyson, K. R., Elkins, C., and de Silva, A. M. (2008). A novel mechanism of complement inhibition unmasked by a tick salivary protein that binds to properdin. J. Immunol. 180, 3964-3968. doi: 10.4049/jimmunol.180.6.3964

Valdés, J. J. (2014). Antihistamine response: a dynamically refined function at the host-tick interface. Parasit. Vectors 7:491. doi: 10.1186/s13071-014-0491-9

Valdés, J. J., Cabezas-Cruz, A., Sima, R., Butterill, P. T., Rùžek, D., and Nuttall, P. A. (2016). Substrate prediction of Ixodes ricinus salivary lipocalins differentially expressed during Borrelia afzelii infection. Sci. Rep. 6:32372. doi: $10.1038 /$ srep32372

Valenzuela, J. G., Charlab, R., Mather, T. N., and Ribeiro, J. M. (2000). Purification, cloning, and expression of a novel salivary anticomplement protein from the tick, Ixodes scapularis. J. Biol. Chem. 275, 18717-18723. doi: 10.1074/jbc.M001486200

Villar, M., Ayllón, N., Alberdi, P., Moreno, A., Moreno, M., Tobes, R., et al. (2015a). Integrated metabolomics, transcriptomics and proteomics identifies metabolic pathways affected by Anaplasma phagocytophilum infection in tick cells. Mol. Cell. Proteomics 14, 3154-3172. doi: 10.1074/mcp.M115.051938
Villar, M., Ayllón, N., Kocan, K. M., Bonzón-Kulichenko, E., Alberdi, P., Blouin, E. F., et al. (2015b). Identification and characterization of Anaplasma phagocytophilum proteins involved in infection of the tick vector, Ixodes scapularis. PLoS ONE 10:e0137237. doi: 10.1371/journal.pone.01 37237

Villar, M., López, V., Ayllón, N., Cabezas-Cruz, A., López, J. A., Vázquez, J., et al. (2016). The intracellular bacterium Anaplasma phagocytophilum selectively manipulates the levels of vertebrate host proteins in the tick vector Ixodes scapularis. Parasit. Vectors 9:467. doi: 10.1186/s13071-0161747-3

Villar, M., Marina, A., and de la Fuente, J. (2017). Applying proteomics to tick vaccine development: where are we? Expert Rev. Proteomics 14, 211-221. doi: 10.1080/14789450.2017.1284590

Villar, M., Popara, M., Ayllón, N., Fernández de Mera, I. G., Mateos-Hernández, L., Galindo, R. C., et al. (2014). A systems biology approach to the characterization of stress response in Dermacentor reticulatus tick unfed larvae. PLoS ONE 9:e89564. doi: 10.1371/journal.pone.0089564

Wagemakers, A., Coumou, J., Schuijt, T. J., Oei, A., Nijhof, A. M., van 't Veer, C., et al. (2016). An Ixodes ricinus tick salivary lectin pathway inhibitor protects Borrelia burgdorferi sensu lato from human complement. Vector Borne Zoonotic Dis. 16, 223-228. doi: 10.1089/vbz.2015. 1901

Weisheit, S., Villar, M., Tykalová, H., Popara, M., Loecherbach, J., Watson, M., et al. (2015). Ixodes scapularis and Ixodes ricinus tick cell lines respond to infection with tick-borne encephalitis virus: transcriptomic and proteomic analysis. Parasit. Vectors 8:599. doi: 10.1186/s13071-015-1210-x

Wikel, S. K., and Allen, J. R. (1977). Acquired resistance to ticks. III. Cobra venom factor and the resistance response. Immunology 32, 457-465.

Conflict of Interest Statement: The authors declare that the research was conducted in the absence of any commercial or financial relationships that could be construed as a potential conflict of interest.

Copyright (c) 2017 Contreras, Alberdi, Fernández De Mera, Krull, Nijhof, Villar and De La Fuente. This is an open-access article distributed under the terms of the Creative Commons Attribution License (CC BY). The use, distribution or reproduction in other forums is permitted, provided the original author (s) or licensor are credited and that the original publication in this journal is cited, in accordance with accepted academic practice. No use, distribution or reproduction is permitted which does not comply with these terms. 